\title{
JUDGING THE TRUTH-VALUE OF A STATEMENT IN AND OUT OF A DEEP PROCESSING CONTEXT
}

\author{
Teresa Garcia-Marques \\ William James Center for Research, ISPA-Instituto Universitário \\ Rita R. Silva \\ Social Cognition Center Cologne, University of Cologne \\ Joana Mello \\ William James Center for Research, ISPA-Instituto Universitário
}

\begin{abstract}
Individuals judge fluently processed statements truer than disfluent statements, which reflects an illusion of truth. A dual-processing approach to the truth effect suggests that cognitive resources and motivation for accuracy should moderate this effect. However, previous research has only manipulated participants' capacity during the encoding of the statements. We directly test an account of the truth effect based on processing features of System 1, manipulating both capacity and motivation at the time of the truth judgments. Fluency was manipulated either through repetition (Experiment 1) or perceptual contrast (Experiment 2). Congruent with dualistic assumptions, the evaluations of participants in the high-capacity and highmotivation conditions differed from those of participants in the other three conditions. However, the pattern of the truth effect in that specific condition was not the same in both experiments: in Experiment 1, the illusion of truth was only reduced, whereas the effect was completely reversed in Experiment 2.
\end{abstract}

Keywords: illusion of truth, dual-processes, motivation, cognitive resources

The illusion of truth effect shows that repeated statements are evaluated as more probably true than new statements (e.g., Bacon, 1979; Begg, Armour, \& Kerr, 1985;

\footnotetext{
The writing of this article was supported by the Research and Development Project grant PTDC/ PSI-PCO/121916/2010, awarded to Teresa Garcia-Marques and UID/PSI/04810/2013 Science and Technology grant by the Portuguese Foundation for Science and Technology.

Correspondence concerning this article should be addressed to Teresa Garcia-Marques, ISPA- Instituto Universitário, Rua Jardim do Tabaco, 34, 1149-041 Lisboa, Portugal; E-mail: gmarques@ispa.pt 
Hasher, Goldstein, \& Toppino, 1977). This highly reliable effect (see Dechêne, Stahl, Hansen, \& Wänke, 2010 for a meta-analysis) seems to be associated with a System 1 type of processing ecology (e.g., Sloman, 1996) in which truth judgments are made on the basis of our intuitions, as well as cues such as how easily/fluently we process information. However, the assumption that fluency-based truth judgments are associated with System 1 lacks direct support. System 1 processing operations do not depend on the availability of cognitive and motivational resources (e.g., Smith \& DeCoster, 2000). To our knowledge, no experiment has ever directly manipulated the processing conditions under which illusions of truth occur, at least not in a way that is fully congruent to what is assumed by dual-process theories (for a review, see Chaiken \& Trope, 1999).

In this article, we provide direct empirical evidence for the theoretical assumption that processing fluency effects on truth judgments are associated with System 1, which shows for the first time that individuals' capacity and motivation resources at the time of a truth judgment moderate the effect. The relevance of this approach goes beyond simply offering empirical support to something that authors already assume to know. We believe that by clarifying which processing conditions favor and which ones oppose illusions of truth we can better understand how and when the different components involved in the truth effect (e.g., Unkelbach \& Stahl, 2009) exert their effects.

\section{THE ILLUSION OF TRUTH EFFECT}

The illusion of truth effect shows that the experience of processing a repeated statement increases its perceived truth-value (e.g., Bacon, 1979; Hasher et al., 1977). The truth effect has gathered quite a considerable amount of evidence since Hasher and colleagues (1977) first demonstrated it (see the meta-analytic review by Dechêne et al., 2010). Because "repetition is an illogical basis for truth" (Begg, Anas, \& Farinacci, 1992, p. 446), as simply repeating a claim does not bring further factual support for it, many authors have been interested in understanding what processes lie behind this effect of repetition and why it informs us about the validity of information.

There is a general consensus among the different perspectives of the truth effect that the subjective experience of fluency elicited by repetition plays an important role in the emergence of the effect (e.g., Begg et al., 1992). Processing fluency is assumed to provide an automatic input for judgments of truth (i.e., a process that occurs without awareness and that is uncontrollable and effortless; see Bargh, 1989). Since its origin, the truth effect has been associated with memory, under the assumption that information stored in memory is perceived as knowledge. Thus, in line with the dual-process approach to memory (Jacoby, 1991), fluency's use in supporting truth judgments has been seen as evidence for an automatic component of memory (Begg et al., 1992). In line with this approach, the truth effect is understood as an implicit memory phenomenon; the subjective experience of familiarity is not only attributed to previous presentation but also (mis)attributed to 
truth (e.g., Begg et al., 1992; Hawkins \& Hoch, 1992). This reasoning suggests that if we understand and become aware of the correct source of our feeling of familiarity, we may prevent its misattribution to truth.

Some authors have challenged the memory explanation for illusions of truth, assuming that the truth effect simply describes a well-learned association between processing fluency and truth (e.g., Reber \& Unkelbach, 2010; Unkelbach, 2007). According to this approach, fluency offers a direct path for inferring the truthvalue of a statement with no need for implicit memory mechanisms. Supporting this assumption, researchers have shown that mere manipulations of perceptual fluency (with no link to memory) promote effects similar to those of repetition and familiarity (e.g., Koch \& Forgas, 2012; Reber \& Schwarz, 1999; Unkelbach, 2007), even though the strength of the effect is usually smaller than the one obtained from familiarity (for a discussion, see Parks \& Toth, 2006; Silva \& Garcia-Marques, 2011; Silva, Garcia-Marques, \& Mello, 2015).

It is important to our claim that all the theoretical perspectives of the truth effect assume that it relies on cognitive processing features that oppose the use of propositional thinking and careful analysis of any information received. It does not matter whether the effect is perceived as an implicit memory phenomenon (e.g., Begg et al., 1992), a bias exerted by processing fluency (e.g., Reber \& Schwarz, 1999), or an associative learning process (e.g., Unkelbach, 2007). All of these mechanisms are associated with a superficial type of processing that does not depend on individuals' motivations or cognitive resources (for reviews see Chaiken \& Trope, 1999; Smith \& DeCoster, 2000). This suggests that processing conditions that favor a deeper and more careful analysis of information (high motivation and high capacity) may hamper illusions of truth. In fact, this is what a dual-process account of the truth effect would predict, even if that account is independent of memory mechanisms.

\section{THE DUAL-PROCESS ACCOUNT OF THE ILLUSION OF TRUTH EFFECT}

A dual-process account of the truth effect assumes that the fluency pathway to truth is supported by System 1 operations (a less analytic system that is fast, automatic, and independent of cognitive resources and/or motivation; see, for example, Smith \& DeCoster, 2000). System 2 (a more analytic system that is slower and depends on the availability of both cognitive resources and motivation; see, for example, Smith \& DeCoster, 2000) is supposed to rely on other judgment-relevant cues under favorable processing conditions (i.e., the simultaneous presence of cognitive resources and motivation for processing). Several experiments have shown that manipulations of both motivation and capacity affect the degree to which individuals elaborate on information (for reviews, see Chaiken \& Trope, 1999; Smith \& DeCoster, 2000). More specifically, the lack of either cognitive resources or motivation will prevent individuals from deeply and carefully processing information (see Smith, 1994). So having no cognitive resources, no motivation, or none of the two will lead individuals to engage in a superficial mode of processing (i.e., Sys- 
tem 1). Research also shows that having both motivation and capacity is necessary to, for example, elaborate on the content of persuasive messages (instead of using more peripheral cues; see Petty \& Cacioppo, 1986), individuate a given person (instead of relying on stereotypes; see, for example, Fiske \& Neuberg, 1990), make more rational decisions (e.g., overcoming bias, Kahneman, 2003), and develop logical thinking (e.g., Kirkpatrick \& Epstein, 1992).

It seems to us that many, if not all, theoretical approaches of the truth effect either implicitly or explicitly share dual-process assumptions (at the very least by contrasting automatic and controlled processes, e.g., Begg et al., 1992). There is some evidence supporting this observation; for example, studies that created conditions favoring low levels of learning during the familiarization phase with the statements showed that illusions of truth emerged independent of conscious explicit retrieval processes (e.g., Hawkins \& Hoch, 1992; Hawkins, Hoch, \& Meyers-Levy, 2001). Perhaps the most paradigmatic evidence of a dual-processing perspective of the truth effect, however, is the study by Begg et al. (1992) which used the process dissociation procedure (PDP; Jacoby, 1991). Their results show that the magnitude of the effect increases when individuals' attentions are divided during familiarization (encoding) with statements, and that the controlled, explicit memory component is reduced.

However, a careful analysis of these studies shows that they only indirectly support the idea that illusions of truth are more likely to occur in a System 1 type of ecology. This is because these empirical approaches focused on participants' processing conditions in the first phase of the experimental procedure (i.e., familiarization with the statements) and not in the second phase, when truth judgments are in fact made. By reducing the levels of learning during the stage of familiarization with the statements, this necessarily interferes with participants' ability to rely on explicit memory (and thus on some features of System 2 type of processing) in the second phase (truth evaluation). Individuals simply do not have the "resources" to rely on explicit memory because the information was never processed deeply in the first place. As a result, whereas these empirical strategies are useful for isolating illusions of truth from explicit memory processes, they become less adequate for understanding the interplay between System 1 and System 2 in producing the effect. To understand the interplay between the two systems' processing features on the truth effect itself (and not memory's role in it), it is preferable to use manipulations that only distinguish between System 1 and System 2 at the moment when truth judgments are made. Few experiments have done this, and those that have only did so indirectly, either by including older adults in the samples (e.g., Skurnik, Yoon, Park, \& Schwarz, 2005) or delaying the interval between the familiarization and truth evaluation phases (e.g., Bacon, 1979; Brown \& Nix, 1996; Garcia-Marques, Silva, Reber, \& Unkelbach, 2015; Hasher et al., 1977; Hawkins \& Hoch, 1992; Skurnik et al., 2005). These studies also showed that illusions of truth are not dependent on explicit memory because delaying the truth evaluation up to a month (e.g., Brown \& Nix, 1996) did not eliminate the effect; in some cases, it even boosted it (e.g., Hawkins \& Hoch, 1992). This evidence suggests that reducing processing capacity favors the emergence of illusions of truth. 
To our knowledge, no empirical approach has ever addressed the specific processing conditions during the judgment phase that may reduce (or increase) the effect, that is, the availability (or lack) of motivation and cognitive resources for processing information at the moment that the truth evaluations are provided. As stated above, results in the literature fit with the assumption that illusions of truth rely on automatic processes, but no direct data support the idea that a more analytic processing mode would overcome the effect. In addition, to our knowledge there is no empirical evidence clarifying the role that task engagement has in the emergence of illusions of truth; we do not know if participants in previous studies were highly engaged in the truth judgment task or not. In our view, they probably were not because the literature on dual processes suggests that highly engaged individuals do not exclusively rely on fluency to make their judgments (see Chaiken \& Trope, 1999). Accordingly, under conditions that favor deeper processing (System 2), i.e., high capacity associated with high motivation, individuals may rely on other judgment-relevant cues that go beyond the fast-acting subjective experience of fluency (one being explicit memory, but see Unkelbach \& Stahl, 2009 for the different components of the truth effect). As a result, we expect that illusions of truth will be attenuated or will even disappear under these processing conditions. Consequently, participants' levels of capacity and motivation at the time of truth judgment (independent of previous levels of learning) should moderate the magnitude of the truth effect.

\section{CURRENT EXPERIMENTS}

We present two experiments that replicate the basic paradigm used to study illusions of truth but with four different processing conditions defined by the orthogonal manipulation of participants' capacities (low vs. high) and motivation levels (low vs. high). In both experiments, processing conditions were manipulated at the time that truth judgments were performed (the second phase of the paradigm). Capacity was manipulated through cognitive overload by asking participants to either keep in mind a sequence of letters while making truth judgments (overload condition) or to write it down immediately beforehand (no-overload condition). Motivation was manipulated by asking participants to make either spontaneous (low motivation) or careful truth evaluations (high motivation). In Experiment 1, these manipulations were introduced within the typical illusion of truth paradigm, using repetition to induce fluency. In Experiment 2, fluency was manipulated by varying the perceptual contrast in which statements were presented.

If the truth effect is a product of the System 1 type of processing and is opposed by System 2, we should expect that the conditions favoring deeper processing (i.e., when individuals have both high motivation and high capacity) will reduce illusions of truth, presumably because they activate other pathways for deciding about truth besides the more automatic fluency-based response. 


\section{EXPERIMENT 1}

In Experiment 1, we tested the hypothesis that participants' motivation and capacity levels moderate the illusion of truth effects, manipulating statements' familiarity level through previous exposure (e.g., Bacon, 1979). Given the high number of replications of repetition-based truth effects and their moderate magnitude (see Dechêne et al., 2010), we expected to replicate the effect in all four motivation $\times$ capacity processing conditions. However, we also expected the effect to be reduced when deeper processing was favored (i.e., when participants have both high capacity and high motivation to elaborate on the statements they are judging).

\section{METHOD}

Participants and Design. The participants were 113 undergraduates (62 women; $M_{\text {age }}=23.00, S D=2.99$ ) who volunteered for the experiment. Participants were randomly assigned to the conditions of a 2 (motivation: spontaneous vs. careful evaluation $) \times 2$ (capacity: overload vs. no-overload $) \times 4$ (versions of material $) \times 2$ (repetition: repeated vs. new statements) mixed-factorial design, with the last factor using a within-participants design.

Material. A set of statements similar to the ones used by Bacon (1979) was created and pre-tested for neutrality regarding truth. Statements were considered neutral if the mean validity ratings of their true and false versions did not differ from one another $(t<1)$ and ranged between $M=3.00$ and $M=5.00$ (rating scale from 1 $=$ "definitely false" to $7=$ "definitely true"). Two primary lists of statements, A1 and B1, were created by randomly selecting 26 different statements (half true and half false) for each of them. Two other lists were composed (A2 and B2) as mirror versions of the previous ones (e.g., if A1 contained the true statement "Crocodiles sleep with their eyes closed," the false version "Crocodiles sleep with their eyes open" was included in A2; see Garcia-Marques et al., 2015).

Procedure. Participants were tested in groups of 15-20. They received a booklet that randomly attributed one experimental condition to them. The first page's instructions, which described the collaboration in three phases, were read aloud by the experimenter. Participants were told that they were going to participate in a series of pre-tests for material that would be used in future experiments. The first task was the evaluation of the interestingness (on a 7-point scale) of a set of statements. Participants were informed that half of the statements were true, and half were false. Thirty statements (the first four statements were rehearsal trials) were then presented by a recorded tape at the rate of one statement every 10 seconds. Approximately a quarter of the participants heard one of the four lists of 26 statements, which were counterbalanced across conditions.

After finishing this task, the experimenter read aloud the instructions for the second phase. This task was to rate the perceived validity (on a 7-point scale: 1 = "sure it's false," 4 = "uncertain," and 7 = "sure it's true") of a set of written statements. In order to "simulate our busy daily lives," a string of eight consonants was written on the blackboard immediately after the participants finished reading the next page of instructions. Participants were instructed to keep the string of consonants 
in their minds until the booklet asked them to report it. They were then asked to read the next page of instructions themselves. These instructions, depending on the individual's experimental condition, either stressed the need to provide their first intuitive judgments regarding the truth-value of each statement, or the importance of making accurate and careful evaluations. After all participants read the motivation instructions, the experimenter wrote a string of eight letters and numbers on the blackboard available in the room and asked participants to memorize it in one minute. After that time had elapsed, the letters and numbers were erased and participants were told to pay attention once again to the instructions in the booklet. For half of the participants, these instructions told them to immediately write down the string of consonants and proceed to the truth ratings task. The other half were told to proceed to the truth evaluation phase, keeping the letters in mind until they had finished; only then were they were asked to write it down. Participants then judged 20 statements, half repeated from the first task (interest ratings) and half new.

\section{RESULTS}

Individual mean truth ratings were analyzed with an ANOVA, entering Repetition as a repeated measures factor, and Motivation and Capacity as between-participants factors. ${ }^{1}$ The results show the presence of illusions of truth, since repeated statements were evaluated truer than new ones $\left(M_{\text {repeated }}=5.73, S E=.08 ; M_{\text {new }}\right.$ $=4.28, S E=.06), F(1,109)=205.84, p<.001, \eta_{\text {partial }}^{2}=.65$. The expected three-way interaction was also significant, suggesting that motivation and capacity interact to produce different levels of the truth effect, $F(1,109)=5.43, p=.020, \eta_{\text {partial }}^{2}=.05$. As Figure 1 shows, the magnitude of the effect was reduced when participants in the no-overload condition were instructed to perform a careful evaluation of the statements. In fact, if we compare the effect in that specific cell of the design (the difference between repeated and new statements, $M_{\text {difference }}=1.03$ ) with all other conditions, the contrast is significant, $t(109)=2.36, p=.020, d=.40$. It explains $76 \%$ of the variability associated with the analysis components of the contrast: not only the variability associated with the three-way interaction but also with all other interactions involving the within-participants factor. Importantly, the associated residual is not significant, $t(109)=1.33, p=.186, d=0.25$, which suggests that, as expected, all other conditions did not differ (the lack of one resource was enough to prevent elaboration). No other reliable effects were found. ${ }^{2}$

1. The factor defined by the versions of material and their counterbalancing did not qualify any of the effects here presented, and was therefore excluded from the analysis.

2. Effects associated with: Motivation, $F(1,109)=1.24 p=.268\left(M_{\text {High }}=5.06, S E=0.07 \mathrm{vs} . M_{\text {Low }}\right.$ $=4.95, S E=0.08)$; Capacity, $F<1\left(M_{\mathrm{High}}=4.95, S E=0.08\right.$ vs. $\left.M_{\text {Low }}=5.05, S E=0.08\right)$; Capacity $\times$ Motivation, $F(1,109)=1.34, p=.248$ (High Motivation: $M_{\text {High }}=4.95, S E=0.10$ vs. $M_{\text {Low }}=5.18, S E=$ 0.10 and Low Motivation: $M_{\text {High }}=4.95, S E=0.12$ vs. $\left.M_{\text {Low }}=4.93, S E=0.11\right)$; Motivation $\times$ Repetition, $F(1,109)=1.62, p=.205$ (High Motivation: $M_{\text {repeated }}=5.85, S E=0.11$ vs. $M_{\text {new }}=4.28, S E=0.08$ and Low Motivation: $M_{\text {repeated }}=5.60, S E=0.13$ vs. $M_{\text {new }}=4.28, S E=0.09$ ); Capacity $\times$ Repetition, $F<1$ (High Capacity: $M_{\text {repeated }}=5.71, S E=.12$ vs. $M_{\text {new }}=4.20, S E=0.09$ and Low Capacity: $M_{\text {repeated }}=5.75, S E=$ 0.12 vs. $\left.M_{\text {new }}=4.36, S E=0.08\right)$. 


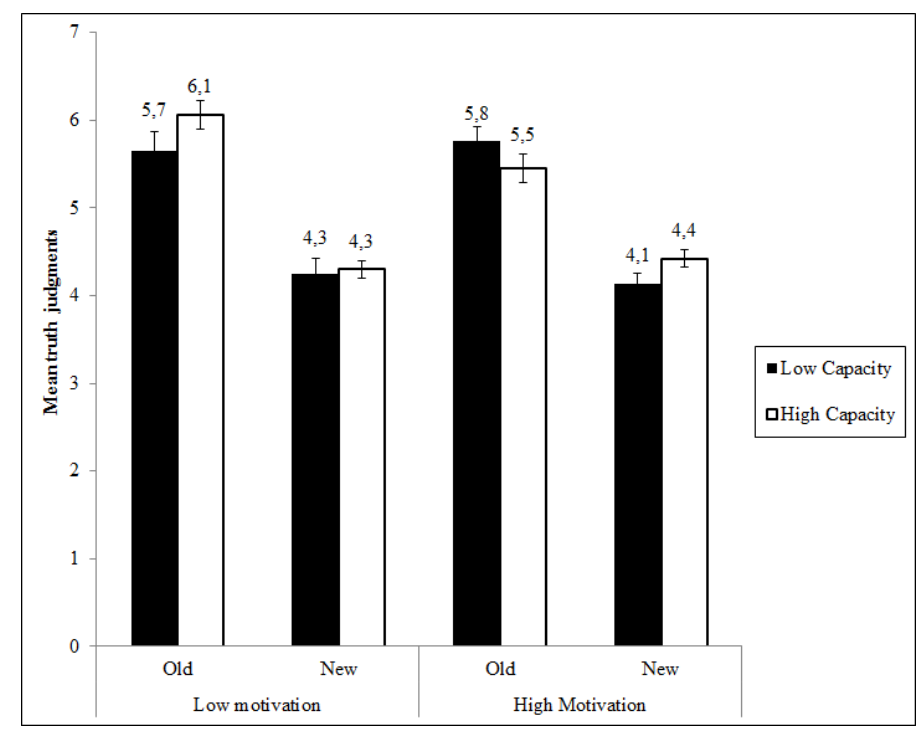

FIGURE 1. Mean ratings of truth to old and new statements, by capacity and motivation condition in Experiment 1. Error bars represent standard errors of the means.

We also categorized participants' responses to each statement as "true" (ratings ranging from 5 to 7), "uncertain" (ratings of 4), and "false" (from 3 to 1) in order to have individual mean proportions of the three types of responses for repeated and new statements. Analyzing this information (categorical) allowed us to understand that the general effect is not only driven by a change in the confidence of evaluations but also by a change in the perceived truth value of the statements. Overall, repeated statements promoted many more "true" judgments than new statements (78\% vs. $44 \%$ ), and much fewer "false" (9\% vs. 23\%) and "uncertain" judgments (13\% vs. $33 \%$ ). As with the previous analysis, the high-capacity and high-motivation condition led to changes in this pattern, which was indicated by the more balanced distribution of "true" and "false" responses to new and old statements in this condition (see Table 1). Furthermore, the proportions of "true" and "false" judgments in this cell were significantly different from the proportions observed in the other three conditions, $t(109)=2.11, p=.038 ; d=.40$ and $t(109)$ $=2.88, p=.005 ; d=.55$ for "true" and "false" responses, respectively. The two contrasts explain a high proportion ( $81 \%$ and $94 \%$, respectively) of the variability associated with the impact of the two factors on the truth effect.

\section{EXPERIMENT 2}

The same hypothesis was tested in the context of a perceptual fluency manipulation. To make the two experiments comparable, participants were also asked to first listen to a set of statements similar to those they would later evaluate for truth. However, all statements were new in the truth ratings phase. Fluency was manipulated through the color-contrast of the statements with the background (e.g., Reber \& Schwarz, 1999; Unkelbach, 2007), which made them either easy or 
TABLE 1. Mean Percentages of Old and New Statements Evaluated As True (Tr), Uncertain (Un), and False (Fa), By Capacity and Motivation Conditions in Experiment 1

\begin{tabular}{|c|c|c|c|c|c|c|c|c|c|c|c|c|}
\hline & \multicolumn{6}{|c|}{ Low Motivation } & \multicolumn{6}{|c|}{ High Motivation } \\
\hline & \multicolumn{3}{|c|}{ Old } & \multicolumn{3}{|c|}{ New } & \multicolumn{3}{|c|}{ Old } & \multicolumn{3}{|c|}{ New } \\
\hline & $\operatorname{Tr}$ & Un & $\mathrm{Fa}$ & $\mathrm{Tr}$ & Un & $\mathrm{Fa}$ & $\operatorname{Tr}$ & Un & $\mathrm{Fa}$ & $\mathrm{Tr}$ & Un & $\mathrm{Fa}$ \\
\hline Low Capacity & 75.8 & 13.8 & 10.4 & 42.3 & 30.1 & 27.6 & 80.5 & 11.9 & 7.6 & 40.9 & 32.4 & 26.7 \\
\hline High Capacity & 85.0 & 10.4 & 4.6 & 45.7 & 33.0 & 21.3 & 70.8 & 17.3 & 11.9 & 46.1 & 35.7 & 18.2 \\
\hline
\end{tabular}

difficult to read. Different from the previous experiment, all the tasks were run on computers, allowing for the collection of response latencies, which are frequently used as an indicator of processing speed (see Reber, Wurtz, \& Zimmermann, 2004).

\section{METHOD}

Participants and Design. Sixty-two female students $\left(M_{\mathrm{age}}=23.43, S D=3.04\right)$ participated in the experiment in exchange for a gift card. Participants were randomly assigned to the conditions of a 2 (motivation: spontaneous vs. careful evaluation) $\times 2$ (capacity: overload vs. no-overload) $\times 2$ (fluency: high vs. low color-contrast) mixed-factorial design, with the last factor using a within-participants design.

Material. The same statements as in Experiment 1 were used. Perceptual fluency was manipulated by presenting statements in colors that were high- and lowcontrast with the white background of the computer screen. Color-contrast was manipulated by varying RGB values, which is a well-established manipulation of perceptual fluency (e.g., Hansen, Dechêne, \& Wänke, 2008; Reber \& Schwarz, 1999; Unkelbach, 2007). Following the combination of values used in previous research (e.g., Hansen et al., 2008; Unkelbach, 2007) we created a low- and high-contrast version of the colors blue (high-contrast: 110, 110, 225; low-contrast: 220, 220, 255) and orange (high-contrast: 242, 144, 0; low-contrast: 255, 255, 175). Statements were written in Arial (size 18) and presented in the center of HP 72 17-inch monitors with $800 \times 600$ pixel resolution against a white background. Participants saw the stimuli from a distance of $\approx 45 \mathrm{~cm}$. and the maximal visual angle of the stimuli was $34.50^{\circ}$ in width and $7.65^{\circ}$ in height.

Procedure. Participants were tested in groups of up to five individuals, who were seated at individual workstations with computers. All instructions were presented on the computer screen. The first general instructions informed participants that they were going to evaluate material for an experiment and that their first task was to listen to a set of statements to get accustomed to the type of material they were going to evaluate. Thirty statements (half true and half false) were then presented aurally through headphones at the pace of one statement every 10 seconds. After presenting all of the statements, the computer program randomly assigned participants to one of the four motivation $\times$ capacity conditions, presenting the exact same instructions provided in Experiment 1. In the truth judgments task, 40 new statements were presented, half in high-contrast colors and the other half in low-contrast. Half of the statements for each level of color-contrast were true, and half were false. Statements were presented one by one, remaining in the 
center of the screen until participants made their evaluations on the same 7-point rating scale as the previous experiment. Both the judgments and the response latencies were recorded.

\section{RESULTS}

Truth ratings were analyzed with an ANOVA with fluency level (color-contrast) as a repeated measures factor. Contrary to what would be predicted if we assumed that perceptual fluency completely replicated repetition, we found no evidence of a main effect for statements' color-contrast on truth ratings $(F<1)$. Both high-contrast and low-contrast statements were generally evaluated as neutral regarding truth $\left(M_{\text {high-contrast }}=4.22, S D=.57 ; M_{\text {low-fluency }}=4.22, S D=.60\right)$. However, a two-way interaction between the motivation and the perceptual fluency factors emerged, $F(1,58)=5.03, p=.028, \eta_{\text {partial }}^{2}=.08$, which suggests that processing features were relevant at the time of judgment. Whereas participants in the low-motivation condition showed the presence of illusions of truth $\left(M_{\text {high-contrast }}=4.21, \mathrm{SD}=.68 ; M_{\text {low- }}\right.$ contrast $=4.09, S D=.71)$, the highly motivated participants did not. In fact, participants in this condition presented a complete reversal of that pattern, evaluating high-fluency statements as less true than low-fluency ones $\left(M_{\text {high-contrast }}=4.23, S D=\right.$ $.44 ; M_{\text {low-contrast }}=4.33, S D=.43$ ). As a result, motivation not only qualified the truth effect promoted by perceptual fluency (as expected), but led individuals to counteract fluency's effect in such a way that the bias was completely reversed.

However, our assumptions also predicted that capacity conditions would qualify illusions of truth so that the effect would be less evident or not present at all for participants with both high motivation and high capacity, even as other conditions would show evidence of the effect. In line with this hypothesis, the expected significant three-way interaction emerged, $F(1,58)=4.61, p=.036 ; \eta_{\text {partial }}^{2}=.08$. As Figure 2 shows, the reversal of the truth effect, $t(58)=2.78, p=.007, d=.74$, found in the high-motivation condition was only clear for participants in the highcapacity condition. In fact, the contrast between the cell in question and the other three, $t(58)=3.21, p=.002, d=.84$, explains $53 \%$ of the variability associated with any interaction between these factors. In addition we found that the typical perceptual fluency effect on truth ratings (the difference between high-fluency and low-fluency statements) was significant for the other three motivation $\times$ capacity conditions, $t(58)=2.03, p=.047, d=.53,{ }^{3}$ which, as expected, did not differ between them $(p>.05)$.

The analysis of participants' response latencies (in ms) allows us to see if: (a) our manipulation of perceptual fluency was effective and if (b) participants in the

3. Effects associated with: Motivation, $F<1\left(M_{\text {High }}=4.28, S E=0.08\right.$ vs. $\left.M_{\text {Low }}=4.24, S E=0.07\right)$; Capacity, $F<1\left(M_{\text {High }}=4.21, S E=0.07\right.$ vs. $\left.M_{\text {Low }}=4.31, S E=0.07\right)$; Capacity $\times$ Motivation, $F(1,109)=$ $1.34, p=.248$ (High Motivation: $M_{\text {High }}=4.28, S E=0.08$ vs. $M_{\text {Low }}=4.24, S E=0.07$ and Low Motivation: $M_{\text {High }}=4.20, S E=0.10$ vs. $\left.M_{\text {Low }}=4.28, S E=0.10\right)$; Capacity $\times$ Repetition, $F(1,57)=1.70, p=.197$ (High Capacity: $M_{\text {high-contrast }}=4.18, S E=0.08$ vs. $M_{\text {low-contrast }}=4.25, S E=0.08$ and Low Capacity: $M_{\text {high-contrast }}=$ $4.35, S E=0.08$ vs. $\left.M_{\text {low-contrast }}=4.28, S E=0.08\right)$. 


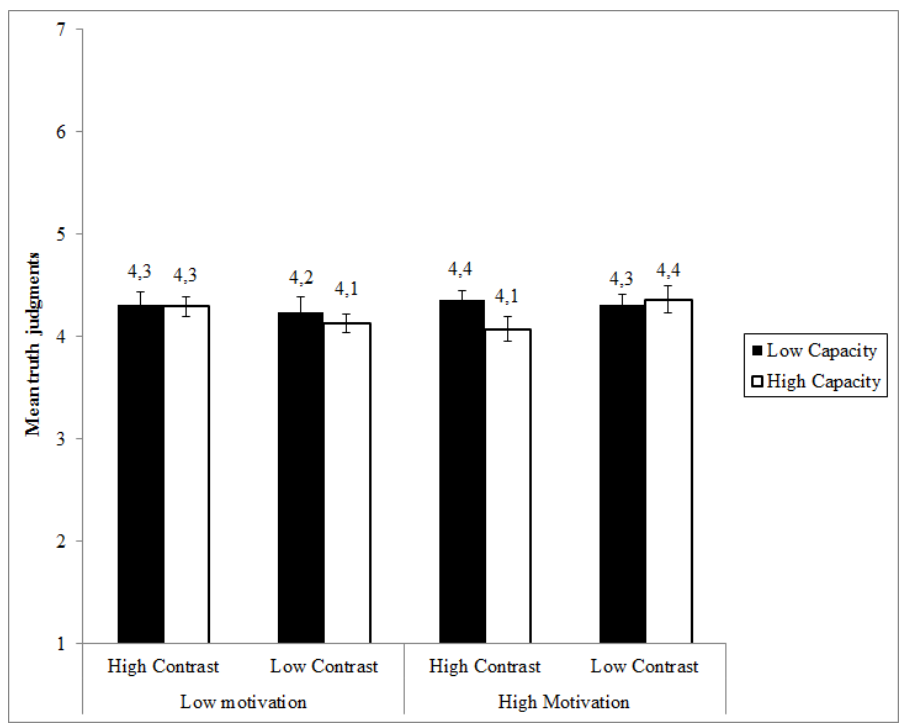

FIGURE 2. Mean ratings of truth to high-contrast and low-contrast statements, by capacity and motivation condition in Experiment 2. Error bars represent standard errors of the means.

target experimental condition (high capacity and high motivation) were engaged in deeper processing of information. Regarding the fluency manipulation, participants were faster to respond to items presented in high-contrast colors $\left(M_{\text {high-contrast }}\right.$ $\left.=7,462, S D=3,326 ; M_{\text {low-contrast }}=8,029, S D=3,411\right), F(1,58)=12.12, p<.001, \eta_{\text {partial }}^{2}=$ .17 , which suggests that high-fluency statements were easier to process than lowfluency statements. The main effect of motivation was that individuals in highmotivation conditions took more time to make their ratings than those in lowmotivation conditions $\left(M_{\text {high-motivation }}=9,666, S D=3,402 ; M_{\text {low-motivation }}=6,059, S D=\right.$ $2,279), F(1,58)=28.94, p<.001, \eta_{\text {partial }}^{2}=.33$, which indicates that the motivation instructions were also effective in producing careful versus spontaneous evaluations, respectively. Regarding the effects of the target experimental condition on response times, the significant interaction between the capacity and motivation factors, $F(1,58)=7.15, p=.010, \eta_{\text {partial }}^{2}=.11$, shows that participants in the highcapacity and high-motivation condition took more time to make their judgments $(M=10,611, S D=3,658)$ than participants in the other three conditions, $t(58)=$ $4.51, p<.001, d=1.18$. In addition, participants in the high-capacity and low-motivation condition were the fastest to provide their ratings $(M=5,124, S D=2,314)$ when compared with participants in the two low-capacity conditions $\left(M_{\text {high-motivation }}\right.$ $\left.=8,783, S D=2,989 ; M_{\text {low-motivation }}=8,939, S D=1,913\right)$. As a result, it seems that participants in the low-capacity conditions used time resources to overcome working memory deficits imposed by the overload manipulation. 


\section{DISCUSSION}

This set of experiments is the first to explicitly and directly manipulate the processing conditions (i.e., capacity and motivation) at the time individuals are making the truth judgments, under which illusions of truth occur. By doing so, it provides the first direct evidence suggesting that the effect is more likely to emerge when people are not scrutinizing statements carefully and when they are engaged in a more automatic and superficial System 1 type of processing. The truth effect was generally replicated when processing fluency was associated with manipulations of familiarity and perceptual contrast. However, as expected, the processing conditions imposed on participants moderated the effect. When participants were motivated and had cognitive resources available, the illusion of truth promoted by repetition was reduced and the illusion of truth promoted by perceptual contrast was reversed. The lack of any of these resources leads to similar results, as would be expected if all these conditions equally prevent elaborative processing. We interpret this as direct evidence that the truth effect is supported by conditions that limit the cognitive resources and/or the motivation for processing information deeply and systematically (that is, conditions that are assumed to favor System 1 processing operations). A deeper and more controlled processing mode (i.e., System 2) will induce responses that go beyond the more intuitive and automatic subjective experience of fluency.

We did not expect the two experiments to reveal different patterns of effects in the high-motivation and high-capacity condition. We simply expected that these conditions would be different from the other capacity $\times$ motivation combinations in both experiments because they would reduce the presence of illusions of truth. However, we believe that the difference between the two experiments is highly informative about the different nature of the processes underlying the effects of repetition and perceptual fluency in judgments of truth. Experiment 2 suggests that individuals reacted as if they had identified and understood that the manipulation of perceptual fluency was a source of bias and for that reason had tried to counteract its effect (so much so that they had even "overcorrected" and reversed it). However, the same thing did not happen in Experiment 1, when the bias associated with repetition was only reduced but not eliminated. Because repetition is a source of both perceptual and conceptual fluency (due to both a verbatim and a semantic repetition; see Garcia-Marques et al., 2015), these different results seem to suggest that, epistemically, conceptual fluency may be perceived as an acceptable and reliable basis for judging truth whereas simple perceptual fluency may not be. This hypothesis may be at the root of why the effects associated with repetition are usually of a higher magnitude than those obtained with perceptual fluency (see Parks \& Toth, 2006; Silva et al., 2015). That is, whereas only participants who are not highly engaged with the task will be biased by perceptual fluency, all participants will be sensitive to repetition effects.

The difference between perceptual fluency and repetition may be informing us that the mechanism behind illusions of truth may not be a simple activation of a well-learned association between fluency and truth that is independent of the 
source of fluency experience (Unkelbach, 2007; Unkelbach \& Stahl, 2009). In addition, the fact that perceptual fluency's effect was actually reversed under high capacity and high motivation seems to fit Reber and Schwarz's (1999) original hypothesis that individuals are biased toward truth when judging statements because perceptually fluent statements feel familiar. This misattribution might then be susceptible to a correction when individuals have motivation and capacity, as is the case with other misattributions (for a review, see Schwarz \& Clore, 1996). Therefore, when elaborating, participants may have "realized" that their judgments were biased due to differences in perceptual fluency, and consequently activated a kind of "skeptical attitude," engaging in an "overcorrection" process. An empirical question is if this "skeptical attitude" is related to a desire to be accurate, which would be more at odds with System 2 types of processing. The results of the high-motivation and high-capacity conditions in Experiment 1 are also not clear in this regard. They suggest that participants rely less exclusively on their experiential feelings, but given that our procedure did not keep track of the true or false status of the statements presented, we cannot say if participants were more accurate in their responses. Future studies should address this issue and clarify whether participants in System 2 processing conditions do correct their biases.

The results of these two experiments allow us to confidently contend that the availability of cognitive resources allied with high motivation for accuracy reduce illusions of truth and that the effect is associated with contexts that fit System 1 type of processing. As our literature review demonstrated, previous studies only provided indirect evidence about this assumption. However, the present experiments furnish clear evidence that individuals are less susceptible to make truth judgments on the basis of fluency under conditions of high motivation and availability of cognitive resources, which lead them to process information more carefully (see also Koch \& Forgas, 2012, for a conceptually similar result with the induction of negative mood and manipulations of perceptual fluency). As a result, it would now be relevant to focus on the relative weight of subjective experiences of fluency that are associated with repetition in conditions where motivation or capacity is lacking. Unkelbach and Stahl (2009) have already provided a framework for this type of question by analyzing the components involved in truth judgments. Some of those components coincide with or support direct fluency effects whereas others can oppose them. A careful systematic manipulation of such components under different motivation $\times$ capacity conditions will help us understand their role.

Last, but not least, the two experiments call attention to the relevance of motivation conditions for the illusion of truth. We have addressed motivation for accuracy that is related to involvement regarding the validity of a position and is likely to make individuals oppose biases present in the environment. However, as Chaiken, Giner-Sorrola, and Chen (1996) suggest, motivation is a variable with many different facets. Defense and impression management motives are not necessarily related to truth and may easily be achieved through biased processing. Additionally, there is evidence (see Arkes, Boehm, \& Xu, 1991; Boehm, 1994) that individual differences, such as the need for cognition (the enjoyment in engaging in effortful analytic processing), have no impact on the magnitude of the illusion 
of truth, which suggests that not all motivational factors are relevant to the disruption of the effect. As a result, if accuracy motives may compel individuals to rely more on other components than on their subjective experiences, other motives or individual characteristics might not, and individuals might rely on their subjective experiences even when their capacity is not restricted. This is another empirical question that deserves attention in the future.

\section{REFERENCES}

Arkes, H. R., Boehm, L. E., \& Xu, G. (1991). Determinants of judged validity. Journal of Experimental Social Psychology, 27, 576605.

Bacon, F. T. (1979). Credibility of repeated statements: Memory for trivia. Journal of Experimental Psychology: Human Learning and Memory, 5, 241-252.

Bargh, J. A. (1989). Conditional automaticity: Varieties of automatic influence in social perception and cognition. In J. S. Uleman \& J. A. Bargh (Eds.), Unintended thought (pp. 3-51). New York: Guilford.

Begg, I. M., Anas, A., \& Farinacci, S. (1992). Dissociation of processes in belief: Source recollection, statement familiarity, and the illusion of truth. Journal of Experimental Psychology: General, 121, 446-458.

Begg, I., Armour, V., \& Kerr, T. (1985). On believing what we remember. Canadian Journal of Behavioural Science, 17, 199-214.

Boehm, L. E. (1994). The validity effect: A search for mediating variables. Personality and Social Psychology Bulletin, 20, 285-293.

Brown, A. S., \& Nix, L. A. (1996). Turning lies into truths: Referential validation of falsehoods. Journal of Experimental Psychology: Learning, Memory, and Cognition, 22, 1088-1100.

Chaiken, S., Giner-Sorolla, R., \& Chen, S. (1996). Beyond accuracy: Defense and impression motives in heuristic and systematic information processing. In P. M. Gollwitzer and J. A. Bargh (Eds.), The psychology of action: Linking motivation and cognition to behavior (pp. 212-252). New York: Guilford.

Chaiken, S., \& Trope, Y. (1999). Dual-process theories in social psychology. New York: Guilford.
Dechêne, A., Stahl, C., Hansen, J., \& Wänke, M. (2010). The truth about the truth: A meta-analytic review of the truth effect. Personality and Social Psychology Review, 14, 238-257.

Fiske, S. T., \& Neuberg, S. L. (1990). A continuum of impression formation, from category-based to individuating processes: Influences of information and motivation on attention and interpretation. Advances in Experimental Social Psychology, 23, 1-74.

Garcia-Marques, T., Silva, R. R., Reber, R., \& Unkelbach, C. (2015). Hearing a statement now and believing the opposite later. Journal of Experimental Social Psychology, 56, 126-129.

Hansen, J., Dechêne, A., \& Wänke, M. (2008). Discrepant fluency increases subjective truth. Journal of Experimental Social Psychology, 44, 687-691.

Hasher, L., Goldstein, D., \& Toppino, T. (1977). Frequency and the conference of referential validity. Journal of Verbal Learning and Verbal Behavior, 16, 107-112.

Hawkins, S. A., \& Hoch, S. J. (1992). Low-involvement learning: Memory without evaluation. Journal of Consumer Research, 19, 212-225.

Hawkins, S. A., Hoch, S. J., \& Meyers-Levy, J. (2001). Low-involvement learning: Repetition and coherence. Journal of Consumer Psychology, 11, 1-11.

Jacoby, L. L. (1991). A process dissociation framework: Separating automatic from intentional uses of memory. Journal of Memory and Language, 30, 513-541.

Kahneman, D. (2003). A perspective on judgment and choice: Mapping bounded rationality. American Psychologist, 58, 697-720. 
Kirkpatrick, L. A., \& Epstein, S. (1992). Cognitive-experiential self-theory and subjective probability: Further evidence for two conceptual systems. Journal of Personality and Social Psychology, 63, 534-544.

Koch, A., \& Forgas, J. P. (2012). Feeling good and feeling truth: The interactive effects of mood and processing fluency on truth judgments. Journal of Experimental Social Psychology, 48, 481-485.

Parks, C. M., \& Toth, J. P. (2006). Fluency, familiarity, aging, and the illusion of truth. Aging, Neuropsychology, and Cognition, 13, 225-253.

Petty, R. E., \& Cacioppo, J. T. (1986). The elaboration likelihood model of persuasion. Advances in Experimental Social Psycholo$g y, 19,123-205$.

Reber, R., \& Schwarz, N. (1999). Effects of perceptual fluency on judgments of truth. Consciousness and Cognition, 8, 338-342.

Reber, R., \& Unkelbach, C. (2010). The epistemic status of processing fluency as source for judgments of truth. Review of Philosophy and Psychology, 1, 563-581.

Reber, R., Wurtz, P., \& Zimmermann, T. D. (2004). Exploring "fringe" consciousness: The subjective experience of perceptual fluency and its objective bases. Consciousness and Cognition, 13, 47-60.

Schwarz, N., \& Clore, G. L. (1996). Feelings and phenomenal experiences. In E. T. Higgins \& A. W. Kruglanski (Eds.), Social psychology: Handbook of basic principles (pp. 433-465). New York: Guilford.

Silva, R. R., \& Garcia-Marques, T. (2011, June). Perceptual and conceptual sources of fluency: Are their signals cognitively distin- guishable? Paper presented at the 16th General Meeting of the European Association of Social Psychology, Stockholm, Sweden.

Silva, R. R., Garcia-Marques, T., \& Mello, J. (in press). The differential effects of fluency due to repetition and fluency due to color contrast on judgments of truth. Psychological Research. Advance online publication.

Skurnik, I., Yoon, C., Park, D. C., \& Schwarz, N. (2005). How warnings about false claims become recommendations. Journal of Consumer Research, 31, 713-724.

Sloman, S. A. (1996). The empirical case for two systems of reasoning. Psychological Bulletin, 119, 3-22.

Smith, E. R. (1994). Procedural knowledge and processing strategies in social cognition. In R. S. Wyer \& T. K. Srull (Eds.) Handbook of social cognition (2nd ed., Vol. 1, pp. 99-151). Hillsdale, NJ: Erlbaum.

Smith, E. R., \& DeCoster, J. (2000). Dual process models in social and cognitive psychology: Conceptual integration and links to underlying memory systems. Personality and Social Psychology Review, 4, 108-131.

Unkelbach, C. (2007). Reversing the truth effect: Learning the interpretation of processing fluency in judgments of truth. Journal of Experimental Psychology: Learning, Memory, and Cognition, 33, 219-230.

Unkelbach, C., \& Stahl, C. (2009). A multinomial modeling approach to dissociate different components of the truth effect. Consciousness and Cognition, 18, 22-38. 